\title{
Linear Lengthening in Iwaidja: an event-quantifying intona- tion at the phonology to semantics/pragmatics interface
}

\author{
Patrick Caudal ${ }^{1}$ and Robert Mailhammer ${ }^{2, *}$
}

1 CNRS \& University of Paris; patrick.caudal@u-paris.fr

2 Western Sydney University; r.mailhammer@westernsydney.edu.au

* Correspondence: patrick.caudal@u-paris.fr

\begin{abstract}
This paper investigates the meaning of a specific intonation contour found in the Northern Australian language Iwaidja called Linear Lengthening Intonation (LLI). Using an experimental field work approach, we analysed approximately 4,000 utterances. We demonstrate that the semantics of LLI is broadly event-quantificational as well as temporally scalar. LLI imposes aspectual selectional restrictions on the verbs it combines with (they must be durative, i.e. cannot describe 'punctual', atomic events), and requires the event description effected by said verbs to exceed a contextually-determined relative scalar meaning (e.g., a 'typical duration' à la (Tatevosov 2008)). Iwaidja differs from other Northern Australian languages with similar intonation patterns (see e.g. (Bishop 2002: 2002; Simard 2013)), in that it does not seem to have any argument NP-related incremental or event scalar meaning. This suggests that LLI is a decidedly grammatical, language-specific device; not a purely iconic kind of expression (even though it also possibly has an iconic dimension).
\end{abstract}

Keywords: Linear Lengthening Intonation - Iwaidja - Australian languages - scalarity - semantics pragmatics - discourse structure

Citation: Caudal, Patrick, and Robert Mailhammer. 2021. Linear

Lengthening in Iwaidja. Languages 6: x. https://doi.org/10.3390/xxxxx

Academic Editor: Firstname Lastname

Received: date

Accepted: date

Published: date

Publisher's Note: MDPI stays neutral with regard to jurisdictional claims in published maps and institutional affiliations.

Copyright: $(12021$ by the authors. Submitted for possible open access publication under the terms and conditions of the Creative Commons Attribution (CC BY) license (https://creativecom-

mons.org/licenses/by/4.0/).

\section{Introduction}

This paper will explore a remarkable intonational contour found in Iwaidja, a severely endangered Indigenous Australian language spoken in the Northwestern Arnhem Land, now mainly on Croker Island (cf. Figure 1). It will be based on data collected in the remote community of Minjilang (Croker Island, N.T.) using experimental elicitation methods as well as more traditional questionnaire-based investigations, over the course of several years (2013-2019). It elaborates on preliminary results presented in (Mailhammer \& Caudal 2019), and will primarily be concerned with the semantics of said contours.
5

6

7

8

9

10

11

12

13

14

15

16 


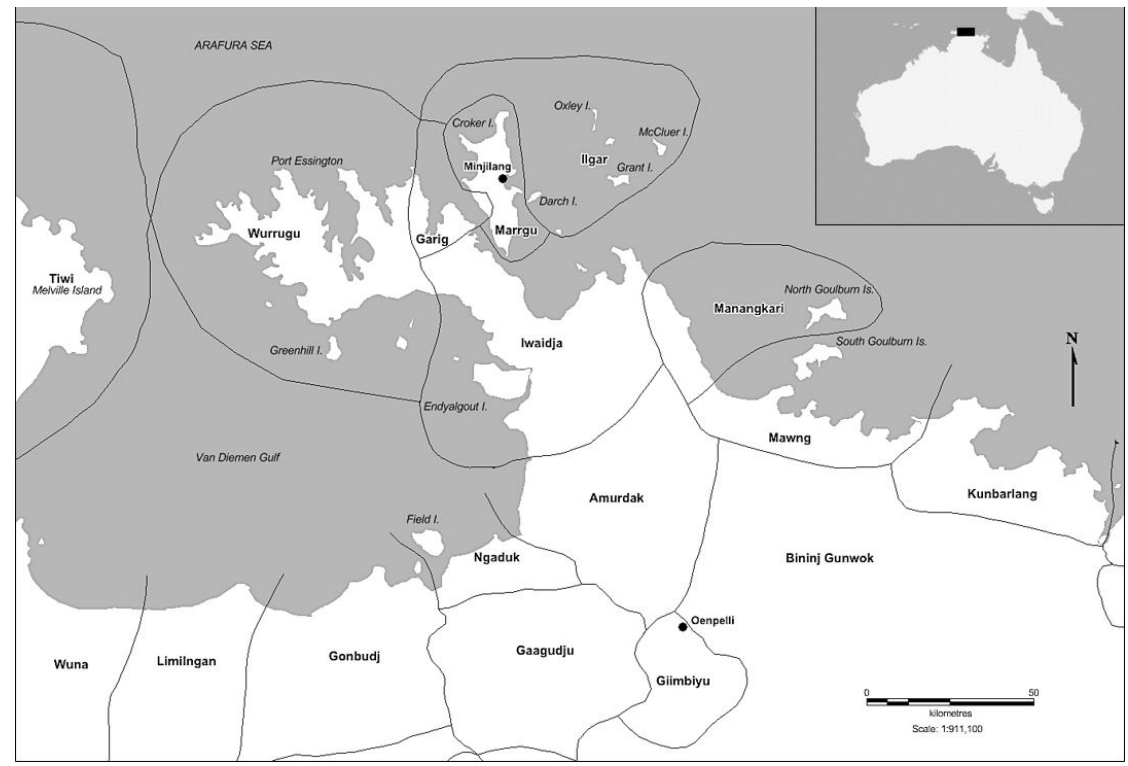

Figure 1. Croker Island and Indigenous languages and their historical locations in Northern Arnhem Land (Mailhammer \& Harvey 2018)

\subsection{Linear Lenghtening Intonation at a glance: formal properties}

Many descriptions of Australian Indigenous languages mention a specific intonation as part of the tune inventory, characterised by a plateau in F0 finishing on a mid to high tone, plus additional lengthening of the last syllable nucleus in the Intonation, (Bishop \& Fletcher 2005: 338), followed by a drop on the next intonation unit. Example (1) illustrates this phenomenon in Iwaidja, the language under scrutiny in this paper, and figure 2 gives a graphic representation generated using Praat (Boersma \& Weenink 2021).

1

(1) Barda r-aka-n

lda jamin::

bartuwa.

(Iwaidja)

Then 3sg.ANT-argue-ANT and 3sg.RECP. EndSequence

'Then they argued for a long while, and (finally) stopped [lit. 'that was it'].

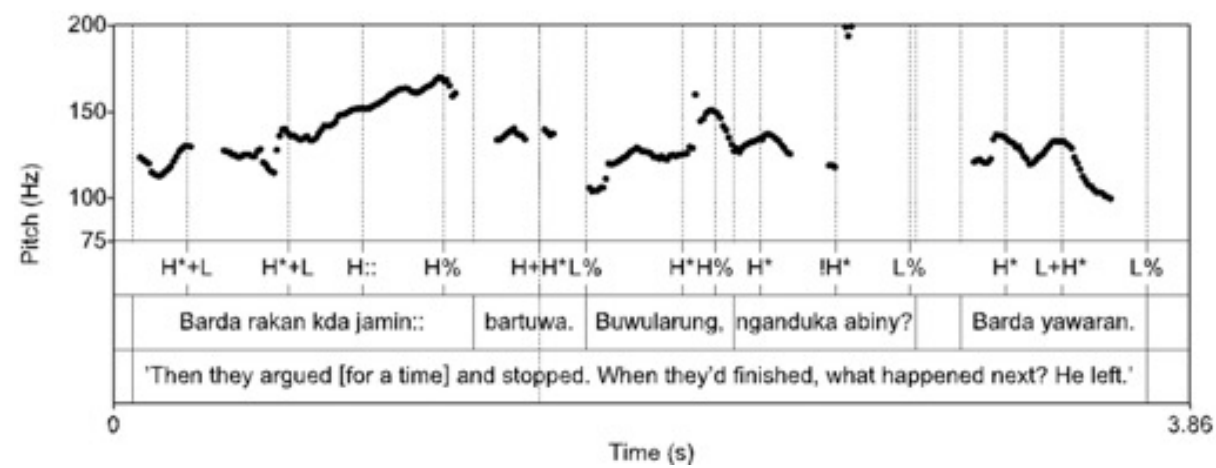

Figure 2. Linear Lengthening Intonation in Iwaidja, from (Mailhammer \& Caudal 2019)

Following (Mailhammer \& Caudal 2019), we will call it Linear Lengthening Intonation (LLI). LLIs are widely attested across Australian languages - cf. (Birch 1999) (Bishop 2002; Simard 2010; Simard 2013; Fletcher 2014). Their formal properties appear reasonably clear, recurrent across all the Australian languages in which it has been documented so far (Bishop 2002; Simard 2013: 67). Lengthening (noted in Figure 2 by the symbol H(:), lengthened high tone) and final high tone at the end of the intonation phrase (noted by $\mathrm{H} \%$, high boundary tone), are the two core criteria for identifying LLI in Iwaidja. Figure 2 demonstrates that at least in this language, Linear Lengthening Intonation does not need not 
show a plateau contour. Although the linear F0 progression can precede the last nucleus by a substantial amount of time, Linear lengthening occurs on the last syllable of a word, which need not be the stressed vowel; see e.g. jamin in Figure 2, a contrastive pronoun used in reciprocal constructions. The following Intonation Phrase can show a falling contour (as in Figure 2); a follow up Intonation Phrase can also be altogether missing, or preceded by a pause of up to ten seconds. By and large, therefore, lengthening appears to demarcate the end of a prosodic unit, possibly an intonational phrase. Note that while we identified examples in our corpus showing not only lengthening of the verb-final vowel, but also of an argument NP, these are clearly marginal. This contrasts with particles-like words such a kirrk 'all/completely', which often appeared with LLI marking in our data but this is hardly surprising, as such particles are sometimes difficult to distinguish from clitics, and are known in Iwaidjan languages to be elements of the extended verb template (Singer 2006: 73-74).

Bona fide LLI formally and semantically contrasts with other prosodic lengthening, notably conveying great distance, as shown in (1). Iwaidja distal deictic baki, 'over there', has a lexicalized, mandatory lengthening attached to it, (speakers rejected made-up examples without lengthening), approximating something like lexical tone (i.e., it contrasts with baki 'tobacco'), (2). Such lengthening is not associated with temporal properties, and cannot be followed by the kind of drop found with LLI. (Bednall 2020b) observes additional formal prosodic differences between 'true' LLI, and (possibly) distance-related prosodic marking in Anindilyakwa - thus, it can occur on the first syllable of a word, unlike LLI, which is always word final. For want of enough data points relating to e.g., spacemeasuring lengthening in Iwaidja, we will leave this issue aside for future research, and focus instead on purely temporal-measuring, bona fide instances of LLI.

(2) Baki::::!

There::::

'Over there, a long way away'

\subsection{Existing analyses and the semantics of Linear Lenghtening Intonation}

In sharp contrast to its formal properties, the semantics of LLI has so far proved elusive (Sharpe 1972; Simard 2013). Existing analyses typically ascribe LLI an iconic status regardless of the language in which it was identified. For instance, (Bishop 2002: 82) claims that it 'dramatizes' the ongoing nature of the action' or 'the extent of some referent' - qua an 'amount of a material substance', or 'extent of a geographical region'. But from a theoretical semantic point of view, what this 'dramatization' function is really about is not very clear; several theoretical concepts come to mind - scalarity in the sense of e.g. (Kennedy 2001), and/or some sort of expressive meaning, which could warrant for instance a multi-dimensional semantic approach à la (Potts 2005; Potts 2007; Gutzmann 2015). Identifying the correct theoretical and formal modelling for the semantics of LLI in Iwaidja will be of central importance to the present account.

It must be stressed that comparative facts alone are sufficient to suggest that the iconic view may not be warranted, as least not in the sense of an on-line, synchronically productive device. Thus in Anindilyakwa, LLI is most commonly borne by a special clitic $=w a$, possibly derived from adverbial ngawa ('still'), cf. (2)-(3) (Bednall 2020a).

(2) nanga-luku-lukwa-marrkaju-wa

d-adz-m-alaka-langwiyu...wa (Anindilyakwa) 3m/3f-RDP-tracks-follow-PAST 3f-f-INALP-foot-ABL.PRG...XTD

3f-tracks-disappear- $\emptyset$

'he kept following her tracks until they disappeared' ['Search' z47-8]

Interestingly, ngawa itself can bear LLI as an isolated word: 
(3)

Engka na-rndarrka. Na-lawurrada (Anindilyakwa)
NEUT.other NEUT/NEUT-grab- $\varnothing$ NEUT-return- $\emptyset \quad n g a .$. wa
ebina-langwiya,
NEUT.that.same-ABL.PRG still...XTD
'It [the she cat] grabbed another one [another kitten], then it brought back, going
along the same way (= all the way back)'. [Bujikeda (Egmond 2012: 220)]

$=w a$ can therefore be regarded as a morphological reflex for LLI - a fact which clearly demonstrates that at least in Anindilyakwa, LLI is not just a an iconic intonational contour with a 'transparent' meaning; it involves an arbitrary form/meaning pairing - in this case, what seems to be a conventionalized construction, involving a specific adverbial in a specific syntactic position. In addition, there seems to be substantial formal variation in the inventory of LLI or LLI-like contours available in each given language - as was notably shown in e.g. (Bishop 2002; Bednall 2020a; Bednall 2020b). As indicated above, and contra e.g. Anindilyakwa and Bininj Gun-Wok, Iwaidja does not seem to licence word initial lengthening. If Bednall's (2020) description of two distinct types of lengthening in Anindilyakwa is correct (i.e., word initial vs. word final lengthening, with respectively temporospatial/emphatic vs. purely temporal meanings), then LLI is just one out of several 'dramatizing' devices in this language. We are definitely dealing in this case with two distinct grammaticalized prosodic markings, with different form-meaning pairings; so that if iconicity can be invoked, it can only be as a diachronic matter, or as an additional, nonnecessary meaning - synchronically, these constitute two distinct conventionalized formmeaning pairings.

\subsection{Background on Iwaidja}

Iwaidja is an under-described, severely endangered non-Pama-Nyungan language pertaining to the Iwaidjan language family. Originally spoken in the Coburg Peninsula area of Northwestern Arnhem Land, it is now one of several Australian Aboriginal languages spoken on Croker Island (with Mawng, Kunwinjku and Kunbarlang being the other major languages spoken on the island; only Mawng and Kunwinjku appear to have long-term viability in the community). Though until very recently, Iwaidja was the main language of the island, deaths of key speakers in the last 15 years, and in general loss of speakers due to non-transmission and migration, have adversely affected the speaker base. It is currently unknown if or to what degree Iwaidja is transmitted to children, and there are probably fewer than 50 proficient Iwaidja speakers currently living on Croker Island. These demographic circumstances preclude access to a large pool of speakers, and therefore render extremely difficult the use of quantitative methods in their standard form. Therefore, the type of experimental work we are reporting on in this paper, is essentially of a qualitative nature (see Mailhammer \& Harvey (2018: 332 for details on the documentary status, the level of analysis and the usage of Iwaidja).

Iwaidja can be described as a weakly polysynthetic language (Fortescue 2016), as its verb template is (i) holophrastic but (ii) does not exhibit productive noun-incorporation. Its verb template only comprises four positions, cf. table 1: the first position is a typical Northern Australian pronominal portmanteau exponent, combining person, gender and number information on the verb's valents, plus some TAM information (TAM $)$, and optionally, deictic information (with a distal vs. proximal distinction). The verb stem occupies the second position, reduplication exponents optionally occupy the third, and another TAM exponent $\left(\mathrm{TAM}_{2}\right)$ occupies the fourth and final position - with $\mathrm{TAM}_{1}$ and $\mathrm{TAM}_{2}$ forming a discontinuous single TAM morph, i.e. some manner of circumfixal morph (this is an instance of so-called distributed exponence à la (Carroll 2016)). 
Table 1. The Iwaidja verb template

\begin{tabular}{|c|c|c|}
\hline 1. Pro.(deixis.)TAM 1 & 2. verb stem & 3. (Reduplication) $4 . \mathrm{TAM}_{2}$ \\
\hline$n g a-$ & ngartbuni & $-\varnothing$ \\
\hline 1sg.PR- & $\begin{array}{l}\text { fall- } \\
\text { 'I fall' }\end{array}$ & $-P R$ \\
\hline
\end{tabular}

Iwaidja possesses two realis/indicative past tense paradigms. The first of these two tenses is an aspectually underspecified anterior tense (ANT), receiving imperfective readings with atelic utterances (except in inchoative, change-of-state contexts, of course), and perfective (or more rarely, perfect) readings with telic and other CoS utterances. The other is a general past imperfective viewpoint (IPFV), with clear pluractional properties as we will see (somewhat reminiscent of imperfective morphology in certain Slavic languages). Given this aspectual partial opposition between the two paradigms, it was highly desirable to control for viewpoint as a key condition of our experiments.

\subsection{Our research question and road-map for the present paper}

The goal we pursue in this paper, is to determine exact semantic import of Iwaidja, and determine what kind of theoretical/formal concepts should be used to account for its alleged 'intensifying' or 'dramatizing' functions.

The main contribution of the paper will be to demonstrate that the semantics of LLI is broadly event-quantificational as well as temporally scalar (in a sense different from event scalarity à la (Kennedy 2012), for its scalar dimension is unrelated to event boundaries and changes-of-state measurement, and rather involves a contextually determined temporal duration scale - and a related standard of comparison). We will propose a formal treatment of this typologically unusual category at the semantics/pragmatics interface, reflecting on what we believe to be a kind of semantic/pragmatic complexity on a par with that of e.g. tenses and temporal discourse connectives. We will specifically argue that it is at once a sentence-level marker, i.e., a VP-modifier constraining the aspectual type of VPdenoted event predicate, and a discourse connective-like item relating two distinct intonation units w.r.t. temporal ordering.

The paper is structured as follows. In section 2, we elaborate on the experimental fieldwork we conducted in order to study LLI-marked event descriptions, using a specially designed databank of video clips to elicit naturalistic event descriptions, combined with targeted, questionnaire-based elicitation, conducted on this typologically unusual, and theoretically challenging intonation in Iwaidja. As we will see, the methodology underlying our experiments rests on a classic two-component model of aspect à la (Smith 1991; Klein 1994), distinguishing between viewpoint aspect (essentially, functions denoted by inflectional verbal morphology, ranging over event predicates denoted by verbs) and event structure aspect (or so-called Aktionsart).

Section 3 discusses the results of our fieldwork; we will notably show that the behaviour of LLI in the data we collected, is consistent with the view that it denotes an event predicate modifier - i.e., something akin to an aspectuo-temporal adverbial. In addition to this, we will also demonstrate that LLIs also behave like discourse-level aspectuo-temporal markers: they often associate with overt discourse connectives (e.g. the bartuwa discourse connective ('and then/that's it'), reinforcing the inherent temporal ordering function of LLIs, and making explicit their interaction with discourse structural parameters. Indeed, LLIs constrain the establishment of discourse relations à la (Asher \& Lascarides 2003); the rising pitch vs. low pitch intonation units involved in a LLI pattern such as fig. 1 must be related by Narration, Result, or exceptionally, Elaboration. These facts reflect, we will argue, on the typologically complex and fine-grained grammar of event descriptions (especially w.r.t. to event duration, closure and ordering) in Iwaidja, and in general, in Australian languages (Caudal 2022a). 
2. Materials and Methods: experimental elicitation of LLI-marked utterances in the field

The data presented below were collected experimentally in the community of Minjilang (Croker Island, N.T., Australia, cf. figure 1), between 2013 and 2018, either by P. Caudal and R. Mailhammer, or by R. Mailhammer alone. They are outcomes of a longterm collaborative project dedicated to the study of the TAM system of Iwaidja, which began with the creation of a dedicated experimental database, namely the Event Description Elicitation Database (EDED).

\subsection{The Event Description Elicitation Database (EDED): constitution and features}

The Event Description Elicitation Database, as the name suggests, was originally devised in order to elicit naturalistic event descriptions in under-documented languages, with complex tense-aspect systems, i.e., combining aspectually meaningful lexical verbs (i.e., endowed with Aktionsart meanings), and aspectually meaningful inflectional markers.

The EDED event type ontology can help elicit both simplex and complex event descriptions. Simplex event types comprise: (a) simple stative, positional stimuli (such as those expressed in English by the positional, stative meanings of sit (as in 'be sitting') or stand (as in 'be standing'); (b) simple activities; (c) simple telic events (both achievements and accomplishments). Complex stimuli include: (a) iterated simplex events, (b) sequences of one or several simplex events, (c) temporal embedding of a simplex, telic event into a complex or simplex event, and $(\mathrm{d})$ even sequences of distinct iterated simplex $(=$ complex) events.

In addition to Aktionsart features, EDED also targets the elicitation of aspectual viewpoint features in the sense of Smith (1991), in combination with all major Aktionsart classes, and including various aspectually coerced readings (de Swart 1998) such as e.g. (i) unfinished, imperfectively viewed accomplishment descriptions (e.g., "Rob was cutting the bread/the tree"), (ii) inchoative states and activities, and (iii) non-culminating/avertive readings (' $\mathrm{X}$ tried and failed to $\mathrm{V} / \mathrm{X}$ nearly $\mathrm{Ved}$ ').

Perfective vs. imperfective viewpoint readings are elicited by means of the visual rendering in the clips of temporal ordering between events - namely strict succession for perfective viewpoint-inducing stimuli, vs. temporal overlap/partial ordering for imperfective viewpoint-inducing films. This corresponds to the now well-known discourse structural effects of aspectual classes of tenses: thus, within a SDRT -based discourse structural approach (Asher \& Lascarides 2003) to context, temporal succession typically associates with the computation of Narration, Occasion, Continuation or Result rhetorical relations, whereas temporal overlap will typically lead to Background rhetorical relations (cf. e.g. (Molendijk 1983; Vet 1980; Lascarides 1992; Asher, Prévot \& Vieu 2007; Caudal 2012).

The EDED database was created over a span of three years: in 2013-2014 by Patrick Caudal and Rob Mailhammer, with additional clips created in 2016 by Patrick Caudal with the assistance of James Bednall. It currently consists of 250+ short video clips, arranged in different experimental protocols. ${ }^{2}$ Each of these protocols respectively comprises between 34 and 83 video clips, and targets the following phenomena:

- Protocol I: interactions between inflectional aspect/viewpoint and event structure

- Protocol II-IV: interactions between tense-aspect information and motion/posture (with sitting, standing, lying, squatting postures, plus itive vs. ventive events being represented in the films)

- $\quad$ Protocol V: event structure, tense-aspect marking, event reduplication/habituality

- Protocol VI: a combination of all the above 


\subsection{Using EDED in the field}

In the field, we used the EDED database with eleven participants, mostly in Minjilang (with one isolated field trip taking place in the community of Warruwi on South Goulburn Island). Nine of these participants were native speakers of Iwaidja ( 5 male, 4 female ranging between 40 and 75 years old). Seven completed the experiment in Iwaidja only; two participants also completed it in English; two participants were proficient speakers of Iwaidja who acquired the language as in late childhood or adults (late bilinguals). They completed the experiment in English only. All participants were shown a series of 34 video clips (List V, 2014); some were shown earlier, longer series I, II, and III (see Appendix A). Our typical recording setup involved one to three informants, and at least two linguists. Informants were shown each of the films sequentially on a computer screen, and were then prompted to described what happened in Iwaidja, using one of three past contexts:

1. simple, non-iterated event description context (' $X$ did $Y$ (once)') (prompt: 'what happened nanguj ['yesterday'] / wularrud ['a long time ago']?')

2. iterated past event description (' $X$ did $Y$ for a long time') (prompt: 'what did he/they keep doing till the sun went down nanguj ['yesterday']?')

3. past habit context ('X used to do $Y^{\prime}$ ) (prompt: 'what did he/they keep on doing all the time wularrud ['a long time ago']?

Their answers were recorded using audio and videorecording equipment, with up to two video recorders (front/back view); see figure 2.

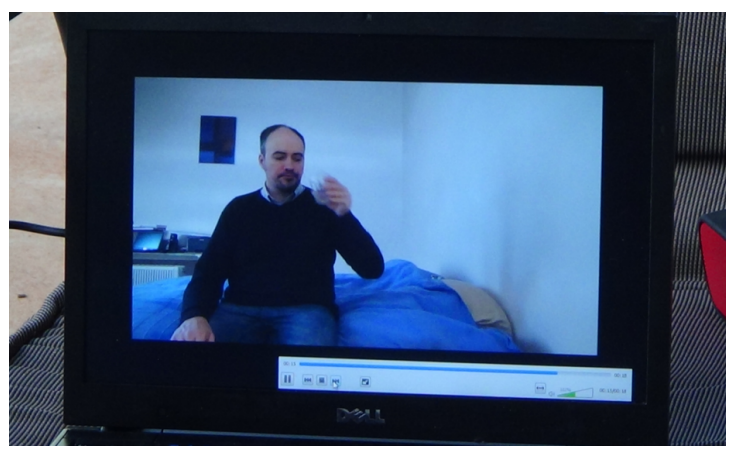

(a)

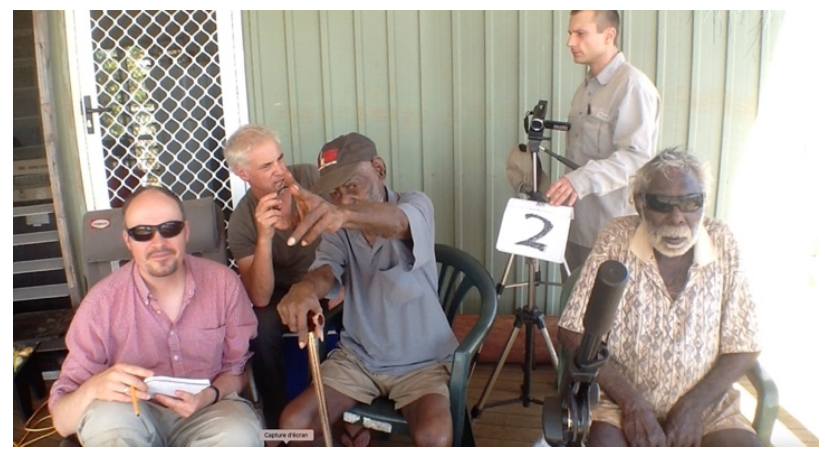

(b)

Figure 3. picture (a) shows a stimulus as seen by the informants, while picture (b) shows a typical set up (with from left to right: linguists Patrick Caudal and Bruce Birch, informant KM, linguist Rob Mailhammer, and informant AB; this photograph was taken on 11. July 2013 at Adjamarduku outstation, Croker Island).

This study and relevant protocols were approved by the Ethics Committee of Western Sydney University (H10237), Chief Investigator Robert Mailhammer.

\section{Results}

Due to the limited number of speakers (by laboratory standards) ${ }^{3}$ we managed to interview, we were unable to construe our fieldwork experiment into a quantitative research project. We collected, transcribed and collected around 4,000 utterances using EDED and complementary questionnaires bearing on a variety of TAM phenomena, only a fraction of which bear LLI marking; the resulting corpus is evidently too limited to warrant the application of efficient quantitative methods.

Although the empirical generalizations we will propose here are based on solid, iterated attestations of certain phenomena, or grammaticality judgments, it is impossible to measure quantitatively what could be coined 'semantic tendencies' of LLI marking, and 
in general rank parameters constraining the interpretation of LLI, in a manner comparable to e.g. (Caudal \& Bednall, this volume). Such a task will have to be differed until substantial further investigations can be conducted in the field.

3.1. The aspectual profile of LLI: event structure selectional restrictions, and distribution with imperfective contexts, posture serial verbs plus reduplication

During our fieldwork elicitation using the EDED database, it quickly appeared that non-durative, punctual telic events tended not to associate with LLI. This was further confirmed by attempts at eliciting acceptability judgements on made up Iwaidja punctual telic utterances bearing LLI marking. Several speakers (KM, CM, JC \& RN) were adamant (6)(7) were impossible; some even questioning the very point of producing such forms.

(6) [Prompt: Would you be happy to say something like *riwukban arlirr:: ? Or karlu? [not]]

JC: Arlarrarr

RM: Uh?? *iwukban::

(arlirr) Why would you want to say that?'

3sg.m.A>3sg.ANT-give-ANT (stick)

'He gave (the stick)::' (TAIM141124JCRNKMededIw_PC, RM: 01:13:13-40)

(7) But you can't say *iwukban::

yawaran. No sense'

3sg3sg.m.A>3sg.ANT-give-ANT 3sg.DIST.ANT-go-ANT

(TAIM141124JCRNKMededIw, RM: 01:14:17-21)

In contrast, LLI can associate with 'durative' telic event descriptions (accomplishments, if you will), cf. (8)-(9) - note that while (8) does not clearly culminate, (9) clearly does; it even incorporates a maximal degree modifier (kirrk 'completely/all') which makes it clear that the maximal degree on the scale associated with the event was reached, and the entirety of the associated incremental theme (the clothing) was hung up. Similarly in (10), the overt quantifier wardad ('one') makes it clear that a single tree is affected, and bartuwa indicates that it was thoroughly and successfully cut. So LLI cannot be described as rejecting telicity, or systematically imposing a detelicizing interpretation with verbs; the non-culmination reading of (8) is contextually determined, but not necessarily prompted by LLI (though it undoubtedly helps in this example). But we will get back to non-culminating readings of LLI-marked verbs below.

(8)

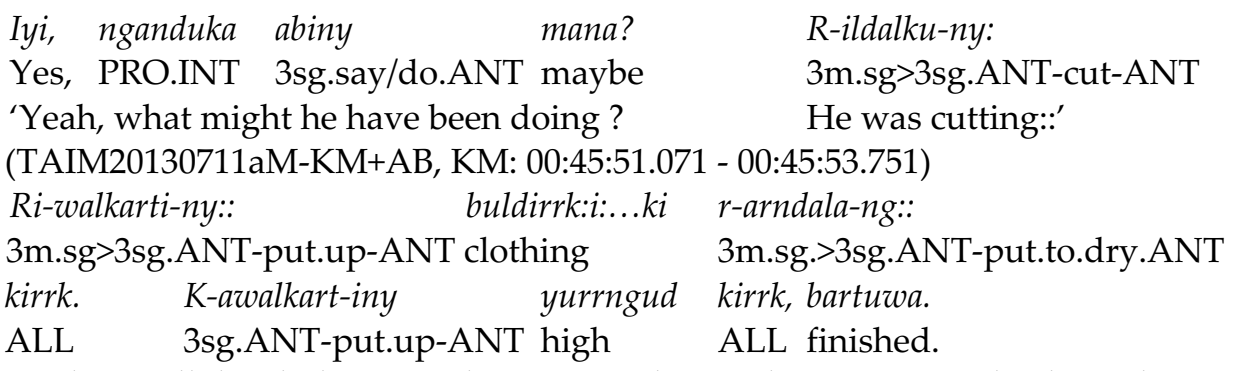

'He hung all the clothing up:: he put it to dry (in the sun):: completely He hung it all up, finished'

(TAIM20130711aM-KM+AB, KM: 00:46:47.318-00:46:52.558)

(10) ildalkuny:

wardad ildalkuny::

bartuwa

3m.sg>3sg.ANT-cut-ANT one 3m.sg>3sg.ANT-cut-ANT finished

"He cut it (long time). One he cut (long time) and that was it'

(TAIM141126ILededIw, IL:00:51:23.800- 00:51:27.525)

This suggests that LLI imposes aspectuo-temporal restrictions on the event predicate conveyed by the verbs. More specifically, it seems to select for durative event predicate, whether telic or atomic, and clearly rejects what can be characterized as atomic telic event 
predicates (cf. (Dowty 1986; Caudal 1999) - i.e. non-scalar punctual event predicates (achievement verbs, if you will). ${ }^{4}$

It does not as a surprise, therefore, that LLI is repeatedly attested in our corpus in combination with markers which suggests a protracted, durative event description. In particular, LLI often pairs up with posture-verb based serial verb constructions (SVCs), cf. (11). As was shown in e.g. (Caudal \& Mailhammer 2017), posture verbs SVCs in Iwaidja contribute an aspectual function whose semantics can be likened to pluractional/iterative/durative aspectual verb 'keep on V-ing' in English. In addition to durative 'keep on' SVCs, LLI can combine with reduplication, either morphological (12) or lexical (13). And LLI can also associate with imperfective morphology on the verb, cf. (13)-(15); and strikingly enough, its semantic effect is then often closer to a Slavic-style imperfective affix, rather than to an average e.g. Romance imperfective viewpoint tense, as it tends then to have pluractional effect, cf. e.g. (13), which spells out through full, lexical reduplication, the pluractional reading of the imperfective form ka-ldalku-ngung. (14) and (15) are also remarkable examples, in that they show a pluractional, atelic reading of a verb, followed up by an expression lexicalizing a successful termination - illustrating a common tendency among Australian languages to treat culmination as a non-lexical issue (cf. (Caudal 2022a) for an extended discussion of this phenomenon).
(11) aringan birdandiny::
yawaran
3sg.IPFV-stand-IPF 3sg.ANT-RED.sing-ANT 3sg.DIST.ANT-go-ANT
'He sang for a long while [lit. 'was standing singing::' ] then he stopped [lit. 'left']'
(TAIM141124JCRNKMededIw_PC.eaf, RM:00:06.20-22)
(12) aringan birdadbirdandiny::
3sg.IPFV-stand-IPFV 3sg.ANT-RED.sing-ANT

'He sang for a long while [lit. 'was standing singing::']

WM+MM-tasc, MM: 00:56:11.380 - 00:56:12.860)

(13) ka-ldalku-ngung:: $\quad k a-w u d b a-n g$

3sg.IPFV-cut-IPFV:: $\quad 3$ sg.ANT-put.down/leave-ANT

ka-ldalku-ny ka-ldalku-ny

3sg.ANT-RED.cut-ANT 3sg.ANT-RED.cut-ANT

\section{(TAIM20130717aW-}

'she was cutting and put it down and cut, cut, cut, cut... and then finished'

(TAIM141126ILededIw, IL: 00:11:16.000-01:11:20.796)

(14) rildalkungung artbung:: bartuwa

3m.sg $>$ 3sg.IPFV-cut-IPFV again:: finished ka-ldalkuny (Iwaidja) 3sg.ANT-RED.cut-ANT

'He kept on cutting it again and again... then he finished'.

(TAIM141126ILededIw, IL 00:10:45.571 - 00:10:48.849)

(15) rimuniny:: barda wurlawu $3 \mathrm{~m} . \mathrm{sg}>3$ sg.ANT-pound-ANT then ready 'He kept pounding it [the food] and after a time it was ready.' (TAIM20130721aMIL+ISL, IL 00:58:13)

Equally unsurprisingly, LLI can freely combine with all types of reduplicated lexically telic verbs, as they describe durative, pluractional events. Examples (16)-(20) were elicited in an explicitly iterative context ('what did he do till sunset?'). Given such a context, both with and without an overt iterative expression (SVC, duration adverbial or expression) LLI is always warranted. Although both SVCs and reduplication are commonly associated to LLI in such contexts, their presence is optional, and only reinforces the markedness of the duration expressed.

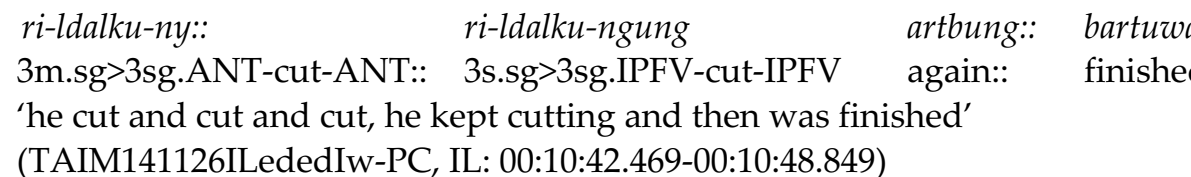

(TAIM141126ILededIw-PC, IL: 00:10:42.469-00:10:48.849) 
(17) nanguj aringan:: k-ardbirru-ny::

yesterday 3sg.IPFV.stand.IPFV 3sg.ANT-throw-ANT

'Yesterday, he kept on throwing (the stone)'

(TAIM141124JCRNKMededIw_PC, JC: 00:46:14.982-00:46:20.000)

(18) aring jurra::

3sg.ANT-stand-ANT 3m.sg $>3$ sg.ANT-stab-ANT paper (bag)

ya-wurryi-ngan manyij

3sg.DIST.IPFV-go.into.water-IPFV sun 411

'He kept on stabbing the paper (bag) as the sun was setting' 412 (TAIM141124JCRNKMededIw_PC, JC: 00:28:40-42) 413

(19) ri-majbunku-ng:: k-artbiru-ny [wardyad]

3sg.m>3sg.IPFV-lift.hold.up-IPFV::: 3sg.ANT-throw-ANT [stone]

[context: slow motion of throwing a stone]

'He was lifting up/holding up [the stone] (then) he threw (it)'

(TAIM141124JCRNKMededIw_PC, RM: 00:45:03-04)

(20) nanguj aringan ri-majbungkungku-ng::

yesterday 3sg.IPFV.stand.IPFV 3sg.IPFV-lift-RED-IPFV

'yesterday, he kept on lifting [that stone]'

(TAIM141124JCRNKMededIw_PC, JC: 00:51:30.058-32.000)

Both ANT and IPFV inflectional marking appear in our data (the latter with a durative, single-event reading (19) or a durative, pluractional reading, as in (16), (18) and (20) - where the presence of reduplication morphology enhances the pluractional reading). Sequence (21) gives two formally different utterances describing the same iterated event, with speaker RM reformulating in the imperfective, a previous utterance in the anterior these two descriptions are therefore truth-conditionally equivalent; this demonstrates the pluractional, durative dimension of the semantics of the Iwaidja imperfective.

(21) JC: nanguj aringan:: kardbirruny::

yesterday 3sg.IPFV-stand-IPFV 3sg.ANT-throw-ANT::

ya-wurryildi-ny manyij

3DIST.ANT-go.down-ANT sun

'Yesterday he kept on throwing [the stone] until the sun went down'

RM: kardbirrukung

3sg.IPFV-throw.RED-IPFV

(TAIM141124JCRNKMededIw_PC 00:46:14-27)

It is also worth observing the reformulation of (18) given in (22), where another speaker immediately describes the same event, but using a different form; the two utterances are given as nearly truth-conditionally equivalent. Starting from the LLI structure in (18), speaker RM paraphrases it in (19) as a markedly emphatic combination of (i) morphological reduplication (rananarkang), (ii) lexical reduplication (ranarkang jurra x 2), (iii) plus temporal ordering (kayrrik 'an then') and event-bounding (burruli 'good/done') expressions - the latter conveying the temporal and discursive function of the low tone, second intonation unit 'closing off' the phonologically complex LLI structure in (18). It is likely that this overtly emphatic reformulation originates in the already substantially emphatic nature of (18) (due to the combination of LLI with a SVC construction).

(22) rananarkang

\begin{tabular}{lllll} 
rananarkang & jurra & \multicolumn{2}{c}{ ranarkang } & jurra \\
3 m.sg $>3$ sg.ANT-RED.stab-ANT & paper & \multicolumn{2}{c}{ 3m.sg $>3$ sg.ANT-stab-ANT } & paper \\
ranarkang & jurra & kayirrk & kuburruburr [???] burruli \\
$3 \mathrm{~m} . \mathrm{sg}>3$ ssg.ANT-stab-ANT & paper & and.then & next.morning good [done]
\end{tabular}


(TAIM20130711aM-KM+AB.eaf, KM: 00:55:58.000 - 00:56:03.305)

\subsection{LLI and aspectually coerced readings of atomic telic verbs}

Interestingly, it turned out that several speakers who had rejected some combinations of LLI with atomic telic verbs, also accepted them with special, marked readings; these uses seem to correspond to cases of aspectual coercion.

First and foremost, we should mention avertive uses (Kuteva 1998; Kuteva et al. 2019) of LLI - where avertive designates a grammatical category covering a wide range of nonculminating meanings. Although the realis past anterior tense (ANT) is used in (24), the result normally associated with the relevant verb is not achieved. (24) closely parallels (25), which has no LLI marking, but bears a modal inflection specifying the 'thwarted' intention underlying the past realis verb '(indeed, the FUT inflection often has volitional or hortative meanings in Iwaidja). We take such examples to be 'non-culminating' in a broad sense: although the target terminus point is reached, the teleologically predicted or desired result state does not come to hold; hence, an incomplete culmination (see (Caudal $2022 b$ ) for an extensive discussion of avertivity and non-culminating telic utterances in the context of Australian languages). (26) further illustrates avertivity in connection with LLI, this time with a 'keep on' construction (waran $(V)$ 'he went on $\left.(\mathrm{V})^{\prime}\right)$, followed by karlu (NEG): the intended goal of the iterative volitional construction with ' go' is thwarted, and the LLI marking contributes to this avertive reading. ${ }^{5}$ Note that (13) is also an instance of avertive reading, albeit of a different kind, aspectually speaking; the event predicate's terminus is not reached in the latter example.

(24) $\quad$ R-urlukba-n:: $\quad$ w-ardajb-ung

3m.sg>3sg.ANT-step.on-ANT:: $\quad 3$ sg.ANT-couldn't.break it-ANT

'He repeatedly tried (= tried hard) to break it with his foot but failed.'

(25) R-urlukba-an, bana-rnukbun.

3m.sg $>3$ sg.ANT-step.on-ANT 3sg.FUT-break-FUT

'He stepped on it trying to break it.'

(TAIM20130711aM-KM+AB, KM: 00:51:54.348 - 00:51:55.468)

(26) W-ara-n:: karlu marukurnaj ri-widari-ny. 
3sg.ANT-go.on-ANT NEG PRO.INDEF 3m.sg>3sgANT-prevent-ANT

'He went on for a while but nothing. Something prevented him from finishing.' (TAIM20130721aM-IL+ISL, ISL: 00:49:34)

After avertive uses, one could mention a range of uses whereby LLI operates at an event's macro-structural level, i.e. at the articulation between sub-events (see (Caudal 2005) for a discussion). Thus, (27) seems to illustrate a situation where LLI bears on the preparatory stage presupposed by the 'come' verb - it indicates that said preparatory stage was protracted.

bingkung kani:: $\quad$ bartuwa
3sg.ANT.came-ANT here-LLI
'he came here slowly and then he was there' (Am_20160609_CMDC_IwAmld,
CM:3:04)

(28)-(29) illustrate a coerced reading, whereby a markedly long interval separates the event's culmination/terminus, from its normally expected result state. It introduces a temporal hiatus between an event's inner stage, and its result stage. This 'hiatus' effect is, to the best of our knowledge, a completely new type of aspectual coercion that has not been documented so far.

(28) $r i-w u-n g:: \varnothing$

k-artbuni-ny

3sg.m>3sg.ANT-hit,kill-ANT

3sg.ANT-fall-ANT

'he hit/killed 3sg. After a while, 3sg. fell'

(Am_20160608_CMDC_LLI, CM: 1:01)

(29) ri-wunbu-ng::

k-artbuni-ny

3sg.m>3sg.ANT-hit.RED-ANT 3sg.ANT-fall-ANT

'he hit 3sg several times [at least twice] and then he fell'

(Am_20160608_CMDC_LLI, CM:1:00)

\subsection{Combination with degree verbs and impact on nominal quantification}

Although we repeatedly tried to elicit LLI marking on scalar verbs, to convey some kind of maximal degree, or marked degree reading, or degree modulation in relation to some incremental theme argument or subject argument, we have systematically failed to find empirical support for the idea that LLI could interact with other measurable dimensions in the semantics of Iwaidja verbs; it seems thoroughly restricted to temporal duration.

Speakers rejected all our attempts at construing such readings, notably in combination with particle kirrk 'completely'. This suggests that if LLI associates with some sort of scalar meaning, it is unrelated to an event's degree scale, and cannot interact with incrementality, or argument distributivity.

Rather, it seems to only relate some utterance to a contextually determined standard of duration - probably on the basis of world-knowledge and specific inferences made in a given context. This does not mean that such readings are impossible for LLI in other languages; but claims made concerning the interaction of LLI with nominal semantics in other languages do not seem to have sound empirical foundations in Iwaidja.

\section{Theoretical discussion and formal analysis of our results}

Let us now turn to the theoretical assessment, and formal modelling of our results. Three salient generalizations uncovered during our field experiments need to be accounted for :

1. LLI expresses subjectively marked durativity 
2. LLI does not seem to relate its evaluative dimension to an event's development per $s e$, nor through an event's degree scale, nor through incrementality nor the internal structure of the denotation of some argument

3. LLI normally rejects atomic telic utterances, but when combined with one, it can give rise to various coerced readings. In addition to this, it can (but need not) have nonculminating/non-resultative interpretative effects

We will try to address these three different properties within a theorical and formal model of LLI in the remainder of this paper.

\subsection{A temporal scalar meaning}

It is quite obvious from the above generalizations, that the semantics of LLI is similar to that of an event description modifier - i.e., some sort of aspectuo-temporal adverbial. As was proposed in (Mailhammer \& Caudal 2019), we will ascribe a tentative type $\langle\langle e, t\rangle,\langle e, t\rangle\rangle$ to its denotation. To be more specific, LLI seems (i) to be restricted to durative events, i.e. to events with a durative run-trace and (b) to convey a relative temporal comparison (in the sense of (Kennedy 2001)), roughly saying that the event at stake has a duration exceeding that normally associated with the relevant event predicate - i.e. what we might want to call a temporal duration standard of comparison.

The idea that event predicates are associated with 'typical duration' is not novel, and has been elaborated upon, and even formalized, in past works, including e.g. (Wyngaerd 2001; Tatevosov 2008; Gyarmathy 2015), a.o. (Tatevosov 2008) proposed to straightforwardly formalize this notion of typical duration (TD) as in (30). Note that we are assuming TD to be contextually evaluated w.r.t. the speaker's current beliefs and knowledge base; it is therefore a subjective, and contextual standard of comparison - not an intersubjective, immutable standard. Tatevosov's definition being extensional, this might prove a problematic - but we will leave the issue of a better definition of TD aside for want of time to address it. ${ }^{6}$

(30) $\operatorname{TD}(\mathrm{P})=\operatorname{mean}\{\mathrm{n} \mid \exists \mathrm{e}[\mathrm{P}(\mathrm{e}) \wedge|\tau(\mathrm{e})|=\mathrm{n}]\}$

We will assume that LLI is associated with a durative zero morph in Iwaidja (morpho-phonologically realized by particle / clitic $=w a$ in Anindilyakwa), effectively an empty clitic attaching to the final syllable of the VP, and (i) causing the linear lengthening of said syllable and (ii) denoting a second order predicate over 'long duration event' predicate Lgdur.

We are formalizing below the semantics of Lgdur within (Asher 2011)'s Type Composition Logic (TCL); as it makes it possible to state in a straightforward way aspectuo-temporal constraints on event types; TCL being endowed with a sortal hierarchy, we will define NON_ATOMIC as the super type encompassing all event types except atomic telic events. $\pi$ is a semantic stack argument ascribed to all predicative types in TCL; all type restrictions borne by a given function are stored in $\pi$, and must be met as the relevant semantic derivation progresses. And when they are met, during e.g., the existential closure of some argument, the relevant restriction is pulled from the stack - or else, if they cannot be met during the relevant functional application, a type mismatch arises. $\pi^{*}$ ARG ${ }_{1}^{\mathrm{P}}$ :NON_ATOMIC indicates that $P^{\prime}$ s first argument (its event variable $e$ ) must be a nonatomic event type; so that when $P$ is bound and the tense operator binds $e$, then if $P$ is an atomic event predicate, then a type mismatch will arise. (Unless coercion bridging functions can apply, and save the semantic derivation day - see below).

To put it in a nutshell, (28) indicates (a) that the predicate at stake must be durative (i.e., it must have a non-atomic run-trace) and (b) that the duration of $P$-events in the context must exceed the typical duration associated with $P$.

$$
\left.\llbracket \text { Lgdur } \rrbracket=\lambda P . L g d u r(P) \leftrightarrow \forall e .\left[P(e)\left(\pi^{*} A R G 1^{P}: N O N \_A T O M I C\right)\right) \wedge \tau(e) \geq T D(P) \wedge \tau(e)<n\right]
$$


Through (31), we are proposing a clearly productive, compositional semantic contribution for LLI, which sets this phenomenon apart from other prosodic markers studied so far w.r.t. their interpretative effects - see e.g. (Portes \& Beyssade 2015), which argue against compositional analyses of other prosodic markers in French.

\subsection{Accounting for 'marked' readings with atomic telic verbs}

As we have seen above, the contribution of $\operatorname{Lgdur}(P)$ is essentially that whatever event $e$ predicate $P$ describe in the current context, must be a non-atomic telic event variable, and that the duration of $\tau(e)$, the run-trace of $e$, must exceeds that of some 'mean' $P$-event $\left.e^{\prime}\right)$. But what if our LLI marker happens bears on an atomic telic verb?

Then two situations can hold: either (i) a 'bridging function' can intervene between Lgdur and $P$, so that an otherwise unwarranted functional application can be salvaged, or (ii) no such bridging function is defined, and the relevant combination is ill-formed.

Following a strategy developed in (Caudal 2020), we will define such 'bridging functions' as lexically conventionalized meaning extensions attached to lexical verbs, or to verb classes at best. (Asher 2011) introduced bridging functions to account for what is generally analyzed as cases of aspectual coercion à la (de Swart 1998). Let us consider (27) again (here repeated as (32)).

$\begin{array}{lll}\text { bingkung } & \text { kani:: } & \text { bartuwa } \\ \text { 3sg.ANT.came-ANT } & \text { here-LLI } & \text { EndSequence. }\end{array}$

'he came here slowly and then he was there' (Am_20160609_CMDC_IwAmld, CM: 3:04)

Come normally conveys an atomic event description, cf. (33):

(33) $\llbracket c o m e^{\prime} \rrbracket=\lambda e \lambda e^{\prime} \lambda x \cdot$ come $^{\prime}\left(\mathrm{e}, \mathrm{x}, \pi^{*} \mathrm{ARG}{ }^{\text {come: }}\right.$ ATOMIC)

As the application of Lgdur to the denotation of come' yields a type mismatch (NON_ATOMIC $\neq$ ATOMIC), only a bridging function can overcome this incompatibility. Fortunately, there exists one, as shown by the 'protracted preparatory stage reading' attached to $(27 / 32)$. We are modelling in (34) a general 'preparatory stage' coercion bridging function $\mathcal{F}_{1}$; the result of its application to come, is the existential binding of the coming event (which becomes a secondary event referent - it is not the main at issue element of the utterance), and the introduction of a non-atomic, unbound preparatory event $\lambda$-variable $e^{\prime}$, which will replace the atomic $e$ (existentially bound in $\mathcal{F}_{1}$, so $\pi^{\prime}$ s initial selectional restriction in (33), namely $\pi^{*} A R G_{1}{ }^{\text {come: }}$ :ATOMIC, is satisfied) as the main contribution of the coerced reading - and is a durative, non-atomic event type, so that it satisfies Lgdur's own selectional restriction in (31) (i.e. $\left.\pi^{*} A R G_{1}^{P}: N O N \_A T O M I C\right)$.

(34) $\mathcal{F}_{1}=\lambda \mathrm{Q} \lambda \mathrm{e}^{\prime} \lambda \mathrm{x} \lambda \pi^{\prime} \lambda \pi . \exists \mathrm{e}\left[\mathrm{Q}(\mathrm{e}: \mathrm{ATOMIC}, \mathrm{x}, \pi) \wedge \varphi \in(\right.$ Non_ATOMic, type $(\mathrm{Q})$, type $(\mathrm{x}))\left(\mathrm{e}^{\prime}, \mathrm{x}, \pi^{\prime}\right) \wedge$ theme $\left.\left(\mathrm{x}_{1}, \mathrm{e}_{1}\right) \wedge \tau\left(\mathrm{e}^{\prime}\right)<{ }^{\circ} \tau(\mathrm{e})\right]$

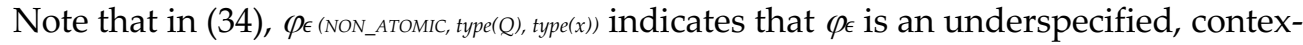
tually determined (preparatory sage) event predicate of NON_ATOMIC (i.e. durative) type, whose sortal type (i.e. lexical event type) can be inferred on the basis of the type of predicate $Q$ and the type of argument $x$. The semantic nature of the underspecified preparatory stage function $\varphi_{\epsilon}$ can be contextually determined using a TCL Glue Logic rule (36), following the general format defined in (35). In the absence of satisfying types in the logical form of an utterance, (36) cannot apply, and $\varphi_{\epsilon}$ remains undefined; we will argue that this results in a degraded acceptability for the coerced reading. 
(35) $(\mathrm{e} \sqsubseteq \mathrm{E} \wedge \mathrm{a} \sqsubseteq \mathrm{A})>\epsilon(\mathrm{e}, \mathrm{a})=\mathrm{P}(\mathrm{e}, \mathrm{a}) \quad$ (with $e$ event type, $a$ object referent type)

663

(36) $(\mathrm{e} \sqsubseteq$ COME $\wedge$ a巨ANIMATE $)>\epsilon(e, a)=$ directed_motion $(e, a)$

Similar bridging functions can be formulated for all the other coerced interpretations identified in §3.2; however, for want of time to formulate them in an appropriate manner, we will leave their formulation to subsequent investigations.

\subsection{On the temporal ordering, discourse-structural effect of LLI}

In addition to its sentence-level, compositional semantic contributions, it appears that LLI is frequently endowed with a discourse structural function. In particular, it predominantly appears in sequence-of-event contexts, and can certainly mark event ordering (although this is not necessarily the case). This quite obvious in its very frequent association with an 'event bounding' expression on its right - qua a post lengthening 'closing off' expression, cf. e.g., bartuwa 'that's it/finished'.

This might seem paradoxical given the durative, and frequently imperfective aspectual semantics of LLI-marked verbs. But as we have seen above, the Iwaidja past imperfective tense often appears to have a pluractional semantics in such contexts (cf. e.g. (16)), rather than a bona fide imperfective viewpoint content in the style of e.g. Romance past imperfectives. And at the end of the day, imperfectivity does not warrant event overlap; it can be associated with sequences-of-events - as is shown by the existence of e.g. 'narrative' uses of various kinds of imperfective tenses, and in general by 'temporal shifts' one can apply to imperfective events as well. See e.g. (Caudal 2022c) for a detailed discussion of such issues in the light of a formal theory of discourse structure.

Following the atter reference, we will propose to ascribe to the semantics of LLI, the ability to convey constraints on possible rhetorical relations attaching whatever expression will appear on the right-hand side of the LLI tune, in its 'drop' component. LLI should in a sense be viewed as coming in two brands: a single intonation-unit-based prosodic marker without any drop component nor event-bounding/temporal ordering function, and double intonation-unit based prosodic marker, with an event bounding. In the latter prosodic construction, the first position corresponds to the discourse referent attached to the LLI-marked VP, and the second to the 'event bounding' expression attached to the tune-dropping intonation unit (cf. e.g., bartuwa in (1)); temporally speaking, the contribution of the second elements of such structure can be likened to a temporal discourse clitic of the 'now/then' type, familiar from several works dedicated to Australian languages see e.g. (Ritz, Dench \& Caudal 2012; Ritz \& Schultze-Berndt 2015; Browne 2020).

Although we will not propose here a detailed formal implementation of the discourse structural function of the event-bounding, event-ordering type of LLIs, we will put forth some analytical, formal suggestions. If we assume a SDRT-style approach of discourse structure à la (Asher \& Lascarides 2003), with the additional technical twist that temporalordering expressions can convey constraints on discourse relations (as proposed in (Caudal 2022c), and that discourse relations can be incorporated in a compositional semantics, then we can formulate the two following very coarse-grained, tentative definitions for the discourse structural semantics of the two intonational components of LLI constructions (high contour + plateau intonation unit in (37), with a durative function meaning, vs. dropping tune intonation unit, with an event ordering function meaning in (38)).

(37) DurativeLLI $=\exists \beta(\beta:[\ldots \mathrm{V} \ldots] \wedge \operatorname{Lgdur}(\mathrm{V}) \wedge ?(\alpha, \beta) \wedge \alpha=$ ?)

(38) BoundingLLI $=\exists \beta$ (Sequence_of_Event_Rel $(\alpha, \beta) \wedge \alpha:[\ldots \mathrm{V} \ldots] \wedge \operatorname{Lgdur}(\mathrm{V}) \wedge \alpha=$ ?])

(37) merely incorporates into an undefined discourse structural function denotation, the $L g d u r$ non-atomic event, durative scalar function already identified above, and applying to the head verbal predicate $\mathrm{V}$ in the DRS dominated by $\beta$. Indeed, ? $\alpha, \beta, \gamma)$ indicates 
that the durative LLI-marked discourse referent $\beta$ can attach to the discourse context at segment $\alpha$, via whatever discourse relation type will be compatible with its semantic profile (we are leaving this as an open question for future investigations).

(38) stipulates that the meaning of the 'dropping tune' LL construction, is to introduce a novel discourse referent $\beta$, by attaching it attach to some segment $\alpha$ previously introduced by the DurativeLLI function (37) (cf. condition $\alpha:[\ldots V \ldots] \wedge L g d u r(V))$. It connects $\beta$ to $\alpha$ via a sequence-of-event inducing discourse relation type Sequence_of_Event_Rel. Discourse relations being binary illocutionary functions between speech act referents, within our TCL-type sortal semantics, they are part of the sortal hierarchy, and Sequence_of_Event_Rel corresponds to the supertype subsuming the Narration, Occasion, Result and Continuation rhetorical functions.

\section{Conclusions}

The main contribution of the paper was to demonstrate that the meaning of LLI is broadly event-quantificational as well as temporally scalar (in a sense different from event scalarity à la (Kennedy 2012). We have seen that its scalar dimension is unrelated to event boundaries and changes-of-state measurement (notably qua incrementality, or so-called event scalarity), and rather involves a contextually determined temporal duration scale - and a related standard of comparison). We have proposed a formal treatment of this typologically unusual category at the semantics/pragmatics interface, reflecting on what we believe to be a kind of semantic/pragmatic complexity on a par with that of e.g., tenses and temporal discourse connectives. We have specifically argued that it is at once a sentencelevel marker, i.e., a VP-modifier constraining the aspectual type of VP-denoted event predicate, and a discourse connective-like item relating two distinct intonation units w.r.t. temporal ordering. Indeed, like aspectual modifiers, LLI imposes aspectual restrictions on the event predicate conveyed by the verb - it selects for durative atelic or telic event predicates (it either rejects or coerces (de Swart 1998) atomic telic event predicates), and it often co-distributes with reduplication (full or partial), imperfective inflectional morphology and associated posture serial verb constructions (Enfield 2002), all of which have clear pluractional/iterative/durative effects in Iwaidja.

But LLIs also behave like discourse-level aspectuo-temporal markers: they often associate with overt discourse connectives (e.g., the bartuwa discourse connective ('and then/that's it'), reinforcing the inherent temporal ordering function of LLIs, and making explicit their interaction with discourse structural parameters. Indeed, LLIs constrain the establishment of discourse relations à la (Asher \& Lascarides 2003); the rising pitch vs. low pitch intonation units involved in a LLI pattern such as fig. 1 must be related by Narration, Result, or exceptionally, Elaboration. These facts reflect, we argue, on the typologically complex and fine-grained grammar of event descriptions (especially w.r.t. to event duration, closure and ordering) in Iwaidja, and in general, in Australian languages.

\section{Patents}

Supplementary Materials: The EDED database is available upon request from the authors.

Author Contributions: Conception of field work experiments and questionnaires: Patrick Caudal and Robert Mailhammer; data collection in the field: Robert Mailhammer, Patrick Caudal (with some assistance from Bruce Birch); writing - original draft preparation, Patrick Caudal; writingreview and editing, Robert Mailhammer; supervision, Robert Mailhammer and Patrick Caudal.; project administration, Patrick Caudal and Robert Mailhammer; funding acquisition, Robert Mailhammer and Patrick Caudal. All authors have read and agreed to the published version of the manuscript.

Funding: This research was funded by the Labex Empirical Foundations of Linguistics (Agence Nationale de la Recherche programme Investissements d'Avenir, ANR-10LABX-0083), subprojects GD4, 
GL3 and MEQTAME (Strands 3 and 2) (CI: Patrick Caudal) (2010-), the CNRS SMI project Complexité morphologique et sémantique de la modalité en Iwaidja (2018-2019) (CI: Patrick Caudal), the CNRS FEMIDAL ('Formal / Experimental Methods and In-depth Description of Australian Indigenous Languages') International Research Project (2021-) (CI: Patrick Caudal), and by a Discovery Grant (DP130103935, CI Robert Mailhammer) by the Australian Research Council. The APC was funded by the Labex EFL (GL3, Strand 3, Project GL3, CI: Patrick Caudal)

Institutional Review Board Statement: The study was conducted according to the guidelines of the Declaration of Helsinki, and approved by the Ethics Committee of Western Sydney University (H10237, 3 October 2013).

Informed Consent Statement: Informed consent was obtained from all subjects involved in the study.

Acknowledgments: We would like to express our heartfelt gratitude to our Iwaidja consultants and teachers for sharing their insights and discussing the data with us as well as for sitting through the experiments. We also thank Bruce Birch for discussions about Iwaidja from which many insights resulted, and for his support of our fieldwork.

Conflicts of Interest: The authors declare no conflict of interest. The funders had no role in the design of the study; in the collection, analyses, or interpretation of data; in the writing of the manuscript, or in the decision to publish the results.
772 


\section{Appendix A}

LIST I

1. Door open

2. Baby sleeping

3. Closed door

4. Arguing1 fades in out

5. Started Turning wheel II

6. Sitting gave axe

7. Started drinking

8. Rob cutting Pat greeted

9. Cutting the tree down

10. Started Running

11. Hanging up washing

12. Sleeping woke up

14. Peeling potato

15. Thinking

16. Cut tree down

17. Argued1 fades in out

18. Sleeping starts crying

19. Cutting wood gave

20. Took bottle

21. Cut tree down saw

22. Cutting bread II

23. Sad

24. Throwing stone imperf

25. Peeled potato

26. Broke bottle

27. Sat Drank Put Down

28. Stood up left

29. Extending arms
UST II

1. Open closed door

2. Baby sleeping

3. Closed door

4. Arguing1 fades in out

5. Started Turning wheel II

6. Sitting gave axe

7. Started drinking

8. Rob cutting Pat greeted

9. Cutting the tree down

10. Started Running

11. Hanging up washing

12. Sleeping woke up

14. Peeling potato

15. Thinking

16. Cut tree down

17. Argued 1 fades in out

18. Sleeping starts crying

19. Cutting wood gave

20. Took bottle

21. Cut tree down saw

22. Cutting bread II

23. Sad

24. Throwing stone imperf

25. Peeled potato

26. Broke bottle

27. Sat Drank Put Down

28. Stood up left

29. Extending arms
LIST III

1. Open closed door

2. Baby sleeping

3. Closed door

4. Arguing1 fades in out

5. Started Turning wheel II

6. Sitting gave axe

7. Started drinking

8. Rob cutting Pat greeted

9. Cutting the tree down

10. Started Running

11. Hanging up washing

12. Sleeping woke up

14. Peeling potato

15. Thinking

16. Cut tree down

17. Argued 1 fades in out

18. Sleeping starts crying

19. Cutting wood gave

20. Took bottle

21. Cut tree down saw

22. Cutting bread II

23. Sad

24. Throwing stone imperf

25. Peeled potato

26. Broke bottle

27. Sat Drank Put Down

28. Stood up left

29. Extending arms
LIST IV

1. Sad

2. Hanging

3. Baby sleeping

4. Closed door

5. Extending arms

6. Open closed door

7. Black then white

8. White then black

9. Singing no posture

10. Coughing

11. Spinning

12. Drinking

13. Blinking

14. Kissed

15. Squating grinding

16. Sing whistle

17. Whistle sing

18. Squating ground scratched

19. Lying ground ate scratched

20. Scratched started singing

21. Squating scratched ground

22. Cooked sang

23. Shook took out bread

24. Sang cooked

25. Draw scratch sing

26. Turned Wheel Looked out

27. Turning looking

28. Slid grinding
List V (19-11-2014)

1. Singing no posture

\section{Kissed}

3. Baby sleeping

4. Sang cooked

5. Extending arms

6. Pierced

7. Sit down sneezes stands up

8. Crouch stand iterated

9. Threw stone

10. Lift crate frustrative

11. Open fridge

12. Open fridge frustrative

13. Hanging up washing interruption

14. Was knocking ran by

15. Stood up jumped sat down

16. Cut bread

17. Push fridge frustrative succeed

18. Push fridge frustrative

19. Broke Stick

20. Rake sweep go

21. Sneezing

\section{Switch on}

23. Cut tree down saw

24. Cutting wood gave

25. Whistled sang whistled sang whistled

26. Eat biscuit

27. Sneezing gave water

28. Switch off light 


\begin{tabular}{|c|c|c|c|}
\hline 30. Baby crying & 30. Baby crying & 30. Baby crying & 29. Scratching sing whistle \\
\hline 31. Spinning & 31. Spinning & 31. Spinning & 30. Started Running \\
\hline 32. Jumping pointed & 32. Jumping pointed & 32. Jumping pointed & 31. Running \\
\hline 33. Running & 33. Running & 33. Running & 32. Cutting branch \\
\hline 34. Cut bread & 34. Cut bread & 34. Cut bread & 33. Cut branch \\
\hline 35. Hanging up washing interruption & 35. Hanging up washing interruption & 35. Hanging up washing interruption & 34. Breaking stick imperfective \\
\hline 36. Baby sleeping kissed & 36. Baby sleeping kissed & 36. Baby sleeping kissed & 35. Broke Stick \\
\hline 37. Cutting tree down saw & 37. Cutting tree down saw & 37. Cutting tree down saw & 36. Receiving \\
\hline 39. Started walking & 39. Started walking & 39. Started walking & 37. Received \\
\hline 40. Breaking stick imperfective & 40. Breaking stick imperfective & 40. Breaking stick imperfective & 38. Was piercing \\
\hline 41. Sleeping & 41. Sleeping & 41. Sleeping & 39. Pierced \\
\hline 42. Shook took out bread & 42. Shook took out bread & 42. Shook took out bread & 40. Throwing stone imperf \\
\hline 43. Coughing & 43. Coughing & 44. Jumped & 41. Threw stone \\
\hline 44. Jumped & 44. Jumped & 46. Sat down fell asleep & 42. Cutting bread II \\
\hline 46. Sat down fell asleep & 46. Sat down fell asleep & 48. Sit down sneezes stands up & 43. Cut bread \\
\hline 47. Jumping CUT BEG & 47. Jumping CUT BEG & 49. Turning looking & 44. Peeling potato \\
\hline 48. Sit down sneezes stands up< & 48. Sit down sneezes stands up< & 50. Walking sat & 45. Peeled potato \\
\hline 49. Turning looking & 49. Turning looking & 51. Turning wheel & 46. Cutting tree down saw \\
\hline 50. Walking sat & 50. Walking sat & 54. Threw stone & 47. Cut tree down saw \\
\hline 51. Turning wheel & 51. Turning wheel & 55. Broke Stick & 48. Sleeping woke up \\
\hline 52. Blinking & 52. Blinking & 56. Threw stone better & 49. Lying grinding jumped \\
\hline 53. Sit down sneezes< & 53. Sit down sneezes< & 57. Turned Wheel Looked out & 50. Cutting wood gave \\
\hline 54. Threw stone & 54. Threw stone & 58. Dug up & 51. Sat Drank Put Down \\
\hline 55. Broke Stick & 55. Broke Stick & 61. Kissed & 52. Sit down sneezes stands up \\
\hline 56. Threw stone better & 56. Threw stone better & 62. Hanging & 53. Walking sat \\
\hline 57. Turned Wheel Looked out & 57. Turned Wheel Looked out & 66. Laughing & 54. Lying eating jumped \\
\hline 58. Dug up & 58. Dug up & 67. Throw stick & 55. Hanging up washing interruption \\
\hline 59. Cutting branch imperfective & 59. Cutting branch imperfective & 68. Cut branch & 56. Sitting grinding gave \\
\hline 60. Drinking & 60. Drinking & 69. Was piercing & 57. Stood up jumped sat down \\
\hline 61. Kissed & 61. Kissed & 70. Cooked sang & 58. Was jumping ran by \\
\hline 62. Hanging & 62. Hanging & 71. Pierced & 59. Stood up knocked sat down \\
\hline 64. Threw stone up & 64. Threw stone up & 72. Scratched started singing & 60. Was knocking ran by \\
\hline 65. Sitting & 65. Sitting & 73. Sang cooked & \\
\hline
\end{tabular}

9. Looking ate biscuit

31. walked sat down slept woke up

\section{Switch on and off}

3. Squating ground scratched

34. Kept dropping stone
65. Sitting
65. Sitting

73. Sang cooked 
66. Laughing

67. Throw stick

68. Cut branch
66. Laughing

67. Throw stick

68. Cut branch

69. Was piercing

70. Cooked sang

71. Pierced

72. Scratched started singing

73. Sang cooked

74. Sang scratched

75. Received

76. Receiving

77. Squating grinding gave

78. Squating grinding

79. Squating ground scratched

80. Squating scratched ground

81. Sitting ate scratched ground

82. Lying grinding jumped

83. Lying ground ate scratched

84. Sitting grinding gave

85. slid grinding

86. Lying eating jumped
74. Sang scratched

75. Received

76. Receiving

77. Squating grinding gave

78. Squating grinding

79. Squating ground scratched

80. Squating scratched ground

81. Sitting ate scratched ground

83. Lying ground ate scratched

84. Sitting grinding gave

85. slid grinding

86. Lying eating jumped

87. Whistle sing

88. Sing whistle

89. Scratching sing whistle

90. Draw scratch sing

Discard 1. Door open 


\section{References}

Asher, Nicholas. 2011. Lexical Meaning in Context: A Web of Words. Cambridge: Cambridge University Press.

Asher, Nicholas \& Alex Lascarides. 2003. Logics of Conversation. Cambridge: Cambridge University Press.

Asher, Nicholas, Laurent Prévot \& Laure Vieu. 2007. Setting the Background in Discourse. Discours 1. http://discours.revues.org/301.

Bednall, James. 2020a. Temporal, aspectual and modal expression in Anindilyakwa, the language of the Groote Eylandt archipelago, Australia. Canberra / Paris: ANU \& Université de Paris PhD Thesis.

Bednall, James. 2020b. Aspectual and discourse-emphatic properties of stylised prosodic lengthening in Anindilyakwa. Presented at the ALS 2020 (2020 Conference of the Australian Linguistic Society), Sydney. https://als.asn.au/Conference/Conference2020.

Bishop, Judith. 2002. Aspects of intonation and prosody in Bininj gun-wok: autosegmental-metrical analysis. Melbourne: The University of Melbourne. http://minerva-access.unimelb.edu.au/handle/11343/39569 (21 November, 2014).

Boersma, Paul \& David Weenink. 2021. PRAAT: Doing phonetics by computer Version 6.2.01, retrieved 17 November 2021. https://www.fon.hum.uva.nl/praat/.

Browne, Mitchell. 2020. Contrast and retroactive implicatures: An analysis of =lku 'now, then' in Warlpiri and Warlmanpa. Australian Journal of Linguistics 40(2). 218-245. https://doi.org/10.1080/07268602.2020.1753651.

Carroll, Matthew Jay. 2016. The Ngkolmpu Language with special reference to distributed exponence. Canberra: Australian National University PhD Thesis. https://openresearch-repository.anu.edu.au/handle/1885/116801 (12 March, 2020).

Caudal, Patrick. 1999. Computational Lexical Semantics Incrementality and the So-Called Punctuality of Events. In Proceedings of the 37th annual meeting of the Association for Computational Linguistics on Computational Linguistics, 497-504. Stroudsburg, PA.: Association for Computational Linguistics. http://clair.eecs.umich.edu/aan/paper.php?paper_id=P99-1064 (14 January, 2016).

Caudal, Patrick. 2005. Stage Structure and Stage Salience for Event Semantics. In Paula Kempchinsky \& Roumyana Slabakova (eds.), Aspectual Inquiries (Studies in Natural Language and Linguistic Theory 62), 239-264. Dordrecht: Springer. https://doi.org/10.1007/1-4020-3033-9_11. http://link.springer.com/chapter/10.1007/1-4020-3033-9_11 (1 March, 2016).

Caudal, Patrick. 2012. Pragmatics. In Robert Binnick (ed.), The Oxford Handbook of Tense and Aspect, 269-305. Oxford / New York: Oxford University Press.

Caudal, Patrick. 2020. Coercion for the ages? A thousand years of parallel inchoative histories for the French passé simple and passé composé. Presented at the LSA 2020 (94th annual meeting of the LSA), “Formal approaches to grammaticalization" Workshop, New Orleans.

Caudal, Patrick. 2022a. Culmination/telicity and event delineation in Australian Languages: phonology, morphosyntax, semantics and pragmatics. In Nora Boneh, Daniel Harbour, Ora Matushansky \& Isabelle Roy (eds.), Building on Babel's Rubble. Saint Denis: Presses Universitaires de Vincennes.

Caudal, Patrick. 2022b. Avertive/frustrative markers in Australian languages: blurring the boundaries between aspectuo-temporal and modal meanings. In Kasia M. Jaszczolt (ed.), Understanding Human Time (Oxford Studies of Time in Language and Thought), 22. Oxford: Oxford University Press.

Caudal, Patrick. 2022c. On so-called 'tense uses' in French as context-sensitive constructions. In Martin Becker \& Jakob Egetenmeyer (eds.), Tense, aspect and discourse structure, 20p. Berlin: De Gruyter.

Caudal, Patrick \& Robert Mailhammer. 2017. Associated motion \& posture in Iwaidja. Presented at the Events \& Space : Associated Motion \& Posture (ESAMP 2017), Paris, University of Chicago Center.

Dowty, David R. 1986. The Effects of Aspectual Class on the Temporal Structure of Discourse: Semantics or Pragmatics? Linguistics and Philosophy 9(1)(1). 37-62.

Egmond, Marie-Elaine van. 2012. Enindhilyakwa phonology, morphosyntax and genetic position. University of Sydney Ph.D. dissertation.

Enfield, Nick J. 2002. Cultural logic and syntactic productivity: Associated posture constructions in Lao. In Nick J. Enfield (ed.), Ethnosyntax: Explorations in culture and grammar, 231-258. Oxford: Oxford University Press.

Fletcher, Janet. 2014. Intonation and prosody in Dalabon. In Sun-Ah Jun (ed.), Prosodic Typology II: The Phonology of Intonation and Phrasing. Oxford: Oxford University Press.

Fortescue, Michael. 2016. Polysynthesis: A Diachronic and Typological Perspective. In Oxford Research Encyclopedia of Linguistics. Oxford: Interactive Factory. http://linguistics.oxfordre.com/view/10.1093/acrefore/9780199384655.001.0001/acrefore9780199384655-e-152 (21 November, 2017).

Gutzmann, Daniel. 2015. Use-Conditional Meaning: Studies in Multidimensional Semantics. Oxford: Oxford University Press.

Gyarmathy, Zsofia. 2015. Achievements, durativity and scales. Berlin: Logos Verlag Berlin GmbH.

Kennedy, Christopher. 2001. Polar Opposition and the Ontology of “Degrees." Linguistics and Philosophy 24(1)(1). 33-70.

Kennedy, Christopher. 2012. The composition of incremental change. In Violeta Demonte \& Louise McNally (eds.), Telicity, Change, and State: A Cross-Categorial View of Event Structure, 103-138. Oxford: Oxford University Press.

Klein, Wolfgang. 1994. Time in Language. London; New York: Routledge.

Kuteva, Tania A. 1998. On Identifying an Evasive Gram: Action Narrowly Averted. Studies in Language 22(1). 113-160. https://doi.org/10.1075/sl.22.1.05kut.

Kuteva, Tania, Bas Aarts, Gergana Popova \& Anvita Abbi. 2019. The grammar of 'non-realization.' Studies in Language 43(4). 850-895. https://doi.org/10.1075/sl.18044.kut. 
Lascarides, Alex. 1992. The Progressive and the Imperfective Paradox. Synthèse 87(3). 401-447.

Mailhammer, Robert \& Patrick Caudal. 2019. Linear Lengthening Intonation in English on Croker Island: identifying substrate origins. JournaLIPP 6. 40-56.

Mailhammer, Robert \& Mark Harvey. 2018. A reconstruction of the Proto-Iwaidjan phoneme system. Australian Journal of Linguistics 38 .

Molendijk, Arie. 1983. Les notions de perfectivité et d'imperfectivité dans l'explication de l'emploi du passé simple et de l'Imparfait. Neophilologus 67. 21-34.

Portes, Cristel \& Claire Beyssade. 2015. Is intonational meaning compositional? Verbum: Analecta Neolatina. Akadémiai Kiadó / Pázmány Péter Catholic University Faculty of Humanities and Social Sciences 37(2). 207-233.

Potts, Christopher. 2005. The Logic of Conventional Implicatures (Oxford Studies in Theoretical Linguistics No. 7). Oxford / New York: Oxford University Press. http://linguistlist.org/pubs/books/get-book.cfm?BookID=14553.

Potts, Christopher. 2007. The expressive dimension. Theoretical Linguistics 33(2). 165-198. https://doi.org/10.1515/TL.2007.011.

Ritz, Marie-Eve, Alan Dench \& Patrick Caudal. 2012. Now or Then? The clitic -rru in Panyjima: Temporal properties in discourse. Australian Journal of Linguistics 32(1). 41-72. https://doi.org/10.1080/07268602.2012.657753.

Ritz, Marie-Eve \& Eva Schultze-Berndt. 2015. Time for a change? The semantics and pragmatics of marking temporal progression in an Australian language. Lingua 166. 1-21. https://doi.org/10.1016/j.lingua.2015.07.007.

Sharpe, Margaret. 1972. Alawa Phonology and Grammar. Canberra: Australian Institute for Aboriginal Studies.

Simard, Candide. 2010. The Prosodic Contours of Jaminjung, a Language of Northern Australia. The University of Manchester PhD Thesis. https://www.escholar.manchester.ac.uk/uk-ac-man-scw:88465 (6 March, 2014).

Simard, Candide. 2013. Prosody and Function of "Iconic Lengthening" in Jaminjung. In Connor Youngberg \& Laura Kipp (eds.), SOAS Working Papers in Linguistics volume 16, 65-77.

Singer, Ruth. 2006. Agreement in Mawng: Productive and lexicalised uses of agreement in an Australian language. Melbourne: University of Melbourne PhD Thesis.

Smith, Carlota. 1991. The Parameter of Aspect. Dordrecht: Kluwer.

Swart, Henriëtte de. 1998. Aspect Shift and Coercion. Natural Language and Linguistic Theory 16(2). $347-385$.

Tatevosov, Sergei. 2008. Subeventual structure and non-culmination. In Olivier Bonami \& Patricia Cabredo Hofherr (eds.), Empirical Issues in Syntax and Semantics 7, 393-422. Paris: Université Paris-Sorbonne. http://www.cssp.cnrs.fr/eiss7/.

Vet, Co. 1980. Temps, aspects et adverbes de temps en francais contemporain : essai de semantique formelle. Genève. Droz.

Wyngaerd, Guido Vanden. 2001. Measuring Events. Language. Linguistic Society of America 77(1). 61-90.

\section{Notes}

1 We use Leipzig Glossing Rules with the following addition: ANT=anterior. We use the standard Australianist practical spelling: $<\mathrm{rr}>=/ \mathrm{r} /,<\mathrm{rd}>=/ \mathrm{r} /,<\mathrm{ld}>=/ l^{\mathrm{r}} /,<\mathrm{rld}>=/ \mathrm{l}^{\mathrm{l}},<\mathrm{rt}>=/ \mathrm{d} /,<\mathrm{rn}>=/ \mathrm{l} /,<$ ny $>=/ \mathrm{n} /($ Iwaidja $)<\mathrm{nj}>=/ \mathrm{n} /($ Anindilyakwa $),<\mathrm{ng}>=/ \mathrm{n} /,<\mathrm{rl}>$ $=/ \mathrm{l},<\mathrm{y}>=/ \mathrm{j} /,<\mathrm{h}>=[\mathrm{u}]$. Voicing in stops is not contrastive. We generally use voiced symbols, except for $/ \mathrm{g} / \mathrm{which}$ is spelt $<\mathrm{k}>$, so that sequences $/ \mathrm{ng} /$ can be easily distinguished orthographically $(<\mathrm{nk}>$ ) from the velar nasal /y/, which is written $<$ ng $>$.

2 The various sets of EDED video clips, alongside with the relevant documentation, are accessible upon requests from the authors. To this day, EDED has been used by over 20 other researchers in the field, in order to elicit naturalistic event descriptions in a variety of languages.

3 Initials of the relevant informants are cited in the examples below, but their names cannot be disclosed here.

4 We are departing from claims earlier made in (Mailhammer \& Caudal 2019), where it was argued that LLI did not tend to associate with accomplishment verbs. A more thorough corpus investigation has proven this generalization to be incorrect.

5 (Caudal 2022b) argues that from an areal typological point of view, reduplication and LLI are common markers for construing avertive readings in numerous Australian languages.

6 Moreover, whether or not the difference between the event's duration, and the standard of comparison determined by (TD) is emphatic, might well be a contextual matter. When the prosody is extremely marked, or when additional durative markers are used (reduplication, SVCs), then the resulting utterance seems to be significantly more durative than the relevant standard of comparison 
- hence a feeling of emphasis. However, we will not attempt to formalize further here this possible context sensitivity, as our data is not sufficient for us to ascertain whether non-emphatic readings of LLI are possible, and if so, in what context they appear. 